\title{
Spin Dynamics of Electrons and Holes in p-Doped InAs/GaAs Quantum Dots
}

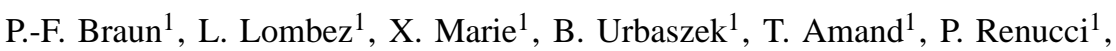 \\ J.-L. Gauffier ${ }^{1}$, V.K. Kalevich ${ }^{2}$, K.V. Kavokin ${ }^{2}$, O. Krebs ${ }^{3}$, and P. Voisin ${ }^{3}$ \\ ${ }^{1}$ LNMO, INSA 135 avenue de Rangueil, 31077 Toulouse Cedex 4, France \\ 2 IOFFE Institute, Politeknicheskaya 26, St-Petersburg 194021, Russia and \\ ${ }^{3}$ Laboratoire de Photonique et Nanostructures, Route de Nozay, 91460 Marcoussis, France
}

Received on 4 April, 2005

\begin{abstract}
We have investigated the electron and hole spin dynamics in p-doped semiconductor InAs/GaAs quantum dots by time resolved photoluminescence. We observe a decay of the average electron spin polarisation down to $1 / 3$ of its initial value with a characteristic time of $T_{\Delta} \approx 500 \mathrm{ps}$. We attribute this decay to the hyperfine interaction of the electron spin with randomly orientated nuclear spins. Magnetic field dependent studies reveal that this efficient spin relaxation mechanism can be suppressed by a field of the order of 100mT. In pump-probe like experiments we demonstrate that the resident hole spin, "written" with a first pulse, remains stable long enough to be "read" $15 \mathrm{~ns}$ later with a second pulse.
\end{abstract}

Keywords: Spin dynamics; InAs/GaAs quantum dots; Time resolved photoluminescence

\section{INTRODUCTION}

Spins of localized electrons in semiconductor quantum dots (QDs) are attractive for future spintronic and quantum information devices since they are not subject to the classical spin relaxation mechanisms known for free carriers [1-5]. Recent theoretical studies have predicted that the dominant mechanism of electron spin relaxation in QDs at low temperature is due to the hyperfine interaction with nuclear spins [6-8]. An electron spin in a quantum dot interacts with a large but finite number of nuclei $N_{L} \approx 10^{3}$ to $10^{5}$ [4]. In the frozen fluctuation model, the sum over the interacting nuclear spins gives rise to a local effective hyperfine field $\mathbf{B}_{N}$ [6]. The electron spin can thus coherently precess around $\mathbf{B}_{N}[6,7]$. However, the amplitude and direction of the effective nuclear field vary strongly from dot to dot. The average electron spin $\langle\mathbf{S}(t)\rangle$ in an ensemble of dots will thus decay as a consequence of the random distribution of the local nuclear effective field. For the sake of simplicity this spin dephasing mechanism of the QD ensemble is termed here "spin relaxation". Note that for repeated measurements on a single QD the hyperfine interaction has the same effect as for an ensemble of dots [6, 7].

The spin dynamics of carriers in III-V or II-VI semiconductor QDs have been studied experimentally by different groups in recent years [9-20]. Spin relaxation times of the neutral exciton longer than 20ns have been found in undoped QDS [13-15]. In n-doped QDs, hole spin relaxation times longer than $10 \mathrm{~ns}$ have been measured $[16,17]$. In all these experiments no manifestation of the electron spin relaxation due to the interaction with nuclei has been observed for the following reasons:

- In undoped QDs the photogenerated electron feels a strong effective magnetic field due to the exchange interaction with the hole [21]. This exchange field is much stronger than the effective hyperfine field of the nuclei, which thus plays a negligible role [22].

- In the experiments performed on n-doped QDs the ground state luminescence corresponds to the radiative recombination of the negatively charged exciton $\mathrm{X}^{-}$formed by one hole, and a pair of electrons with opposite spins in a singlet state [23]. In this case, no effect of the hyperfine interaction with nuclei is expected since the total electron spin in the charged exciton is zero and the hole spin is only weakly coupled to the nuclear spins due to the p-symmetry of the hole Bloch function [24].

The positively charged excitons $\mathrm{X}^{+}$(consisting of one electron and two holes forming a spin singlet) is the ideal configuration to probe the electron spin relaxation mediated by nuclei in QD with optical experiments. The exchange interaction between the electron and the two holes cancels in the $\mathrm{X}^{+}$ground state as in the case of $\mathrm{X}^{-}$. The analysis of the circular polarization of the $\mathrm{X}^{+}$luminescence in $\mathrm{p}$-doped QD following a circularly polarized laser excitation will thus probe directly the spin polarization of the electron. A large spin polarization of the $\mathrm{X}^{+}$luminescence has indeed been observed recently in InAs/GaAs and GaAs/AlGaAs quantum dot photoluminescence (PL) spectra $[25,26]$.

In this paper we present time and polarisation resolved PL experiments in p-doped InAs/GaAs quantum dots. We find that the time dependence of the electron spin polarization exhibits two regimes: the polarization decays within the first 800 ps down to $1 / 3$ of its initial value; then it remains stable with no measurable decay on the radiative lifetime scale. We also show experimentally that this efficient spin relaxation mechanism can be suppressed by the application of a small external magnetic field $(B \approx 100 m T)$. We interpret these results as experimental evidence of electron spin relaxation mediated by the hyperfine interaction with nuclei in an ensemble of QDs [27]. Moreover, in pump-probe like experiments we demonstrate the resident hole spin polarisation stability in our structures over a delay time longer than $15 \mathrm{~ns}$.

\section{SAMPLES AND SETUP}

We have studied three modulation doped QD structures grown by molecular beam epitaxy on (001) GaAs substrates. Very similar results have been obtained for all three samples, which have slightly different doping levels. They con- 
sist of 10 planes of lens shaped self assembled InAs/GaAs QD separated by a $30 \mathrm{~nm}$ GaAs layer; a Beryllium delta doping layer is located $15 \mathrm{~nm}$ below each wetting layer (WL). The nominal acceptor concentration in the presented sample is $N_{A}=15 \times 10^{10} \mathrm{~cm}^{-2}$ per layer. The QD density is about $4 \times 10^{10} \mathrm{~cm}^{-2}$ per plane. The observation of QD ground state PL under strictly resonant excitation (Fig. 1.a) proves that these structures contain on average less than two resident holes on the QD ground state. The samples are excited by $1.5 \mathrm{ps}$ pulses generated by a mode-locked Ti-doped sapphire laser with a repetition frequency of $82 \mathrm{MHz}$. The time resolved PL of the QD ground state is then recorded using a S1 photocathode Streak Camera with an overall time-resolution of 30ps. The excitation pulses are circularly polarized $\left(\sigma^{+}\right)$. The luminescence intensity co-polarized $\left(\mathrm{I}^{+}\right)$and counterpolarized $\left(\mathrm{I}^{-}\right)$with the excitation laser are recorded. The circular polarization degree of the luminescence is defined as $P_{c}=\left(I^{+}-I^{-}\right) /\left(I^{+}+I^{-}\right)$. In the following the arrows $\uparrow, \downarrow$ characterize the spin projection on the $O z$ growth axis of the electron ground states (labelled $S_{c}$ ) whereas $\Uparrow$ and $\Downarrow$ characterise the heavy hole pseudo-spin in the valence ground state (labelled $S_{v}$ ) [18].

\section{SPIN DYNAMICS OF POSITIVELY CHARGED EXCITONS IN INAS/GAAS QUANTUM DOTS}

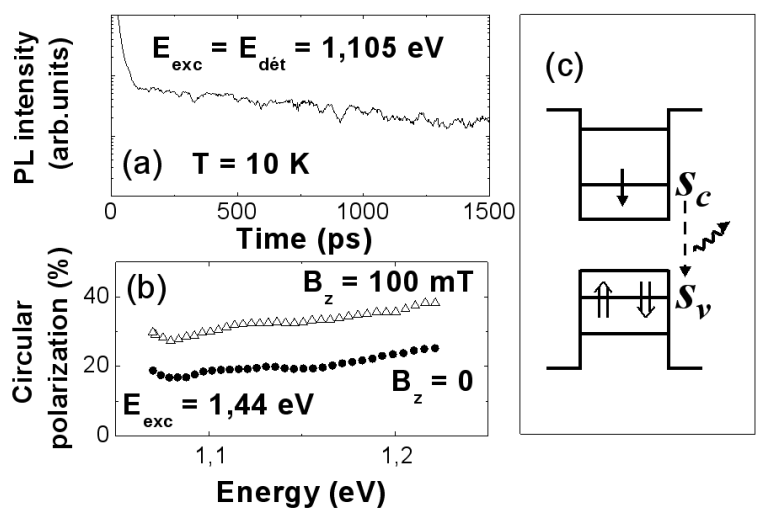

FIG. 1: (a) Intensity of the QD ground state PL for intradot strictly resonant excitation and detection at $1.105 \mathrm{eV}$. Note that the first decay (at $\mathrm{t}<100 \mathrm{ps}$ ) is due to the laser backscattered light. (b) Circular polarization of the QD ground state luminescence for $(\bullet) B_{z}=0$ and $(\triangle) B_{z}=100 \mathrm{mT}$; the excitation energy is $1.44 \mathrm{eV}$, i.e. in the wetting layer (c) Scheme of a positively charged exciton $\mathrm{X}^{+}$formed by a spin polarized electron and two holes with opposite spin angular momentum projection.

The cw PL spectrum of the QD ground states at $\mathrm{T}=10 \mathrm{~K}$ (not shown here) is characterized by a full width at half maximum of about $50 \mathrm{meV}$ due to the fluctuations of size, shape and strain in the ensemble of dots. Fig. 1.b presents the circular polarization of the time integrated PL after a circularly polarized picosecond excitation. The photon excitation en- ergy is tuned to $1.44 \mathrm{eV}$, in the lower part of the WL absorption spectrum. Due to strain and quantum confinement, this absorption energy corresponds to an heavy hole-electron like transition [18]. At zero magnetic field we measure a circular polarization degree of $\approx 19 \%$ of the QD ground state emission. The excitation intensity is about $1 \mathrm{~mW}$; this corresponds to the photogeneration of less than one electron-hole pair per QD. All three p-doped samples that we have studied present circular polarization degrees larger than $10 \%$. In contrast, the same experiment performed in nominally undoped QD samples (not shown here) yields a very small polarization $P_{c}<3 \%$. This weak circular polarization in undoped QD under these non-resonant excitation conditions is a direct consequence of the linearly-polarized neutral exciton eigenstates due to the Anisotropic Exchange Interaction (AEI) between the electron and the hole [13, 14, 28, 29]. The measurement of a significant circular polarization in Fig. 1.b is a strong indication of the successful chemical doping of the QD. For simplicity, we consider for the interpretation that (i) the dots contain a single resident hole and (ii) a single electron-hole pair is optically injected into the dot. Following excitation into the WL, it is well known that the electron spin does not relax during the capture and energy relaxation process in the QD whereas the initial hole spin orientation is lost due to efficient spin relaxation processes in the WL $[18,25,30]$. The recorded PL in the p-doped QD samples corresponds essentially to the radiative recombination of positively charged exciton $\mathrm{X}^{+}$formed with a spin polarized electron and two holes with opposite spin (see Fig. 1.c): $\left|X^{+}\right\rangle=1 / \sqrt{2}(|\Uparrow, \Downarrow, \downarrow\rangle-|\Downarrow, \Uparrow, \downarrow\rangle)$.

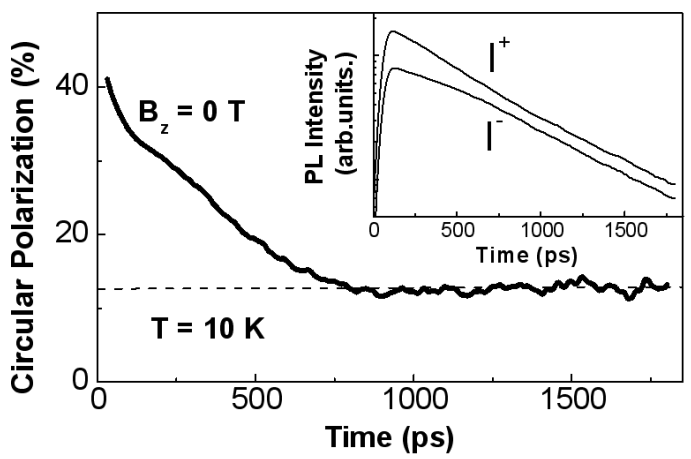

FIG. 2: Circular polarization dynamics of the QD luminescence after a circularly polarized $\sigma^{+}$laser excitation; $E_{\text {exc }} .=1.44 \mathrm{eV}$. Inset: photoluminescence intensity co-polarized $I^{+}$and counter-polarized $I^{-}$with the laser (semi-logarithmic scale). The detection energy is centred at $E_{\text {det }}=1.11 \mathrm{eV}$.

Figure 2 displays the circular polarization dynamics of the QD ground state luminescence (same excitation conditions as Fig.1. b). The inset presents the time evolution of the luminescence intensity components $I^{+}$and $I^{-}$. The circular polarization dynamics in Fig. 2 presents two regimes. The polarization decays within the first 800ps down to a value of about $12 \%$; then it remains stable with no measurable decay on the radiative life-time scale. We can infer that the spin 
relaxation in this second regime is longer than $10 \mathrm{~ns}$. This specific circular polarization dynamics has been observed for any detection energy in the PL spectrum of the QD ground state ensemble. Moreover, we have measured similar kinetics in all the p-doped samples we have studied. All these results are in very good agreement with the predicted electron spin relaxation by nuclei [6-8]. The time dependence of the average electron spin due to the interaction with nuclei can be written as [6]:

$$
\langle\mathbf{S}(t)\rangle=\frac{\mathbf{S}_{0}}{3}\left\{1+2\left[1-2\left(\frac{t}{2 T_{\Delta}}\right)^{2}\right] \exp \left[-\left(\frac{t}{2 T_{\Delta}}\right)^{2}\right]\right\}
$$

where $\mathbf{S}_{0}$ is the initial spin, $T_{\Delta}=\hbar /\left(g_{e} \mu_{B} \Delta_{B}\right)$ is the dephasing time due to the random electron precession frequencies in the randomly distributed frozen fluctuation of the nuclear hyperfine field, $\mu_{B}$ is the Bohr magneton, $g_{e}$ is the electron effective Lande factor. The dispersion of the nuclear hyperfine field $B_{N}$ is described here by a Gaussian distribution characterized by its width $\Delta_{B}: W\left(\mathbf{B}_{N}\right) \approx \exp \left(-\left(\mathbf{B}_{N}\right)^{2} / \Delta_{B}^{2}\right)$ where $\Delta_{B}^{2}=2\left\langle\mathbf{B}_{N}^{2}\right\rangle / 3$ [6]. It is clear from equation (1) that the time dependence of the average electron spin polarization exhibits two regimes. After a strong initial decay with a characteristic time $T_{\Delta}$ the average electron spin polarization is expected to reach a constant value of $1 / 3$ of the initial polarization (Fig. 4) [31]. The circular polarization measured in Fig.1 and Fig. 2 corresponds mainly to the radiative recombination of $\mathrm{X}^{+}$, i.e. it probes directly the spin relaxation of the electron: $P_{c}(t) \approx \rho_{e}(t)=2\langle S(t)\rangle$, where $\rho_{e}$ is the electron spin polarization. The initial value of the average electron spin polarization is here about $\rho_{e}(0) \approx 40 \% ; \rho_{e}(t)$ then drops down to about $1 / 3\left(\rho_{e} \approx 12 \%\right)$ of its initial value in agreement with the predictions of equation (1). After the initial drop the average electron spin polarization remains stable on the radiative lifetime scale. Merkulov et al. calculated that the subsequent electron spin dephasing, which is the result of the variations of the nuclear field direction, occurs on a time scale typically 100 times longer than $T_{\Delta}$ [6]. It can thus not be observed on the radiative lifetime scale.

A key argument for the hyperfine interaction being responsible for the initial polarization decay comes from magnetic field dependent measurements. We have recorded the circular polarization dynamics of the QD ground state luminescence with a magnetic field applied along the $O z$ growth axis. Merkulov et al. and Semenov et al. predict that the electron spin dephasing induced by hyperfine interaction can be strongly suppressed in an external magnetic field $[6,8,32]$. The required magnetic field must be larger than $\Delta_{B}$, which is of the order of $10 m T$ [7], to ensure that the Zeeman interaction of the electron spin with the magnetic field is stronger than the interaction with the nuclei. We see in Fig. 1.b that the timeintegrated circular polarization is almost doubled at the peak of the spectrum when a magnetic field of $B_{z}=100 \mathrm{mT}$ is applied. This strong increase in circular polarization for such a weak external magnetic field is very unusual in non-magnetic semiconductors. Note that the Zeeman splitting energy of the electron in this weak magnetic field is at least 50 times smaller than $k_{B} T$ at $\mathrm{T}=10 \mathrm{~K}$ [21]. Fig. 3 displays the circular polarization dynamics of the QD ground state luminescence with magnetic fields $B_{z}=100 \mathrm{mT}$ and $B_{z}=400 \mathrm{mT}$; the dynamics for $B_{z}=0$ is also presented for comparison. By applying a field of $B_{z}=100 \mathrm{~m} T$ we drastically increase the initial decay time to $\approx 4000 \mathrm{ps}$ as compared to $\approx 500 \mathrm{ps}$ at $B_{z}=0$. This pronounced effect of the small external magnetic field observed in Fig. 3 agrees very well with the expected influence of the external magnetic field on the QD electron spin relaxation induced by nuclei $[6,8]$. The effect observed here is similar to the suppression of the nuclear hyperfine interaction measured recently for localized electrons in lightly doped bulk n-GaAs $[33,34]$.

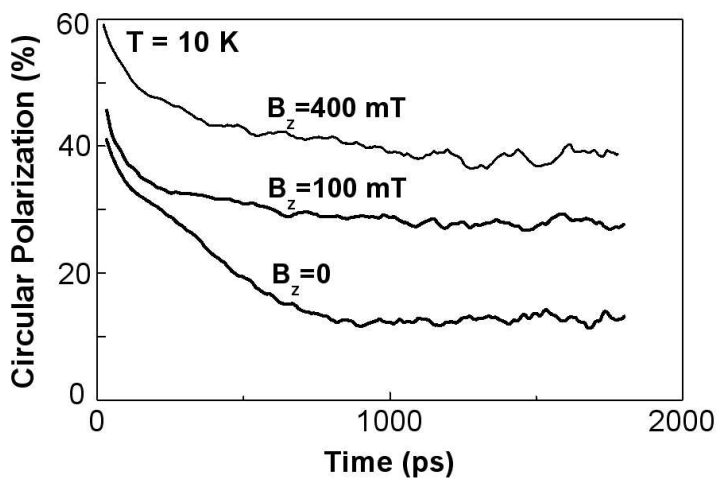

FIG. 3: Circular polarization dynamics of the QD ground state luminescence (semi-log. scale) for $B_{z}=0, B_{z}=100 \mathrm{mT}$ and $B_{z}=400 \mathrm{mT}$.

We see in Fig. 3 that the time evolution of the circular polarization at $B_{z}=400 m T$ is very similar to the behaviour at $B_{z}=100 m T$ [35]. The main difference is a small increase of the initial circular polarization which is probably due to the effect of the magnetic field on the electron spin relaxation during its capture and energy relaxation inside the dot. We still observe a slow initial decay of the circular polarization in Fig. 3 whereas we expect a total suppression of the spin relaxation by nuclei for $B_{z}>100 m T$ (see Fig. 4) [6, 8]. Firstly, we have assumed up to now that the analysed luminescence corresponds only to the radiative recombination of positively charged excitons $\mathrm{X}^{+}$. This is an oversimplified description as neutral excitons $\mathrm{X}^{0}$ or doubly charged excitons $\mathrm{X}^{2+}$ can contribute to the recombination process since some of the dots contain zero or two holes before the optical excitation (the majority of the dots containing one resident hole). The slow initial decay observed in Fig. 3 for $B_{z} \neq 0$ could be due to the complex spin dynamics of $\mathrm{X}^{0}$ or $\mathrm{X}^{2+}$. Secondly, we have neglected in our interpretation the details of the spin-dependent energy relaxation inside the dots. In n-doped InAs/GaAs QDs it has been shown in particular that the strong AEI in the $\mathrm{X}^{-}$ charged exciton hot triplet state plays a significant role in the spin dynamics $[18,36]$. In p-doped QDs a similarly slow spin flip process mediated by the AEI in the $\mathrm{X}^{+}$hot triplet state could occur in QDs where the photogenerated and resident hole spins are parallel. This effect may also contribute to the 
slow initial decay of the circular polarization in Fig. 3 for $B_{z} \neq$ 0 . As the anisotropic exchange energy in the hot triplet state is of the order of $100 \mu \mathrm{eV}$, the suppression of the AEI spin relaxation mechanism would require the application of magnetic fields of the order of a few teslas. Note that in n-doped QD, the application of a field of $B_{z}=100 m T$ does not yield any measurable change in the PL circular polarization dynamics [37].

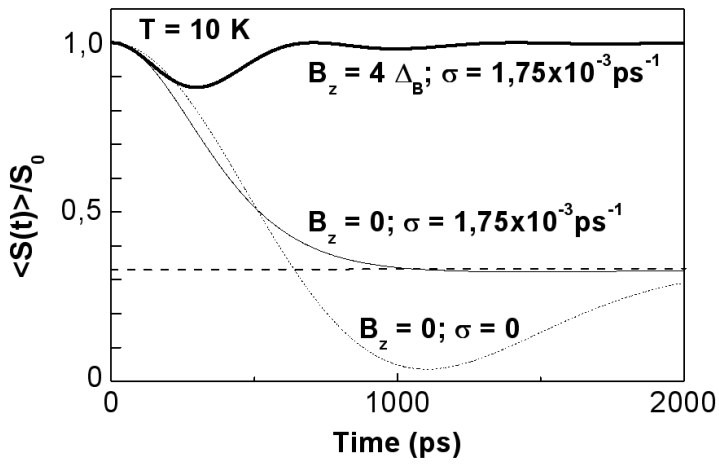

FIG. 4: Calculated time dependence of the average electron spin $<\mathbf{S}(\mathrm{t})>/ \mathbf{S}_{0}$ taking into account the dot size fluctuations via the broadening parameter $\sigma$ (see text).

The initial decay time of the average electron spin polarization due to the interaction with nuclei can be estimated from Fig. $3\left(B_{z}=0\right)$. We find $T_{\Delta} \approx 500$ ps. From Merkulov et al. it can be shown that this dephasing time can be written as:

$$
T_{\Delta}=\hbar\left[n^{2} \sum_{j} I^{j}\left(I^{j}+1\right)\left(A^{j}\right)^{2} /\left(3 N_{L}\right)\right]^{1 / 2}
$$

where $N_{L}$ is the number of nuclei interacting with the electron in the QD, $A^{j}$ the hyperfine constant, $I^{j}$ the spin of the $j^{t h}$ nucleus and $n$ is the number of nuclei per unit cell [6]. The sum goes over all the atoms in the primitive unit cell. We take for the hyperfine constants of As $\left(I^{A s}=3 / 2\right)$ and In $\left(I^{I n}\right.$ =9/2) the values: $A_{A s}=47 \mu \mathrm{eV}$ and $A_{I n}=56 \mu \mathrm{eV}$, consistent with $n=2$ [38]. For a typical dot size (base diameter $\approx 17 \pm$ $3 \mathrm{~nm}$, height $\approx 5 \pm 2 \mathrm{~nm}$ ) [39], we estimate that the number of nuclei in interaction with the electron is $N_{L}=N_{0} \pm \Delta \mathrm{N}_{L} \approx$ $6 \times 10^{4} \pm 4 \times 10^{4}[6,7]$. Equation 2 yields for an InAs dot: $T_{\Delta}^{I n A s} \approx 450 \pm 170 \mathrm{ps}$, in good agreement with the experimental value $(\approx 500 \mathrm{ps})$ if we consider (i) the great uncertainty on the determination of the number of nuclei $N_{L}$ interacting with the electron and (ii) the In/Ga interdiffusion which yields the formation of InGaAs dots rather than pure InAs dots [40]. From the experimental determination of the dephasing time $T_{\Delta}$ we can estimate the dispersion $\Delta_{B}$ of the nuclear hyperfine field $\mathbf{B}_{N}$. We find $\Delta_{B}=\hbar /\left(g_{e} \mu_{B} T_{\Delta}\right) \approx 28 m T$, assuming an electron $g$ factor of $\left|g_{e}\right|=0.8$ as measured by Bayer $e t$ al. [21]. This value of $\Delta_{B}$ is consistent with the effect of the external magnetic field observed in Fig. 1.b and Fig. 3. The external magnetic field $B_{z}=100 \mathrm{mT}$ is about 4 times larger than $\Delta_{B}$. The Fig. 4 presents the calculated average electron spin $\left\langle\mathbf{S}(t)>/ \mathbf{S}_{0}\right.$ for $B_{z}=0$ and $B_{z}=4 \times \Delta_{B}(\approx 100 m T)$ with the parameters $T_{\Delta}=450 \mathrm{ps}$ and $\Delta_{B}=28 m T$ [6].

The calculation of Merkulov et al. (ref. [6]) does not take into account the dot size fluctuations which is a reality in all self-organized QD samples. Clearly, in a quantum dot ensemble, the dot size varies from dot to dot, and consequently the dephasing time $T_{\Delta}$, determined for an ensemble of quantum dots with a given number of interacting nuclei $N_{L}$, will also vary accordingly. We have modified the theory to take into account the dot size variations. It turns out that a convenient analytical approximation can still be obtained when assuming a gaussian broadening on the parameter $T_{\Delta}^{-1}$, according to:

$$
W(\Omega)=\frac{1}{\sqrt{2 \pi} \sigma} \exp \left[-\frac{\left(\Omega-\Omega_{0}\right)^{2}}{2 \sigma^{2}}\right]
$$

where $\Omega=T^{-1}$, and $\Omega_{0}=T_{\Delta}^{-1}$ corresponds to the average dot size $N_{L}$ (this is equivalent to assume a gaussian broadening on $\left.\Delta_{B}=\hbar / g_{e} \mu_{B} T_{\Delta}\right)$. The distribution $W(\Omega)$ corresponds to a distribution of the dot size:

$$
W^{\prime}(N)=\Omega_{0} \frac{N_{0}^{1 / 2}}{2 N^{3 / 2}} W\left[\Omega_{0}\left(\frac{N_{0}}{N}\right)^{1 / 2}\right]
$$

(for $\sigma<<\Omega_{0}$, W'(N) can still be approximated by a gaussian distribution).

The time evolution of the ensemble average spin is then calculated from expression (1) derived from the model of Merkulov et al. and (3), as:

$$
\langle\langle\mathbf{S}(t)\rangle\rangle=\int\langle\mathbf{S}(t)\rangle W(\Omega) d \Omega
$$

After some calculations, the final result is the following:

$$
\begin{gathered}
\langle\langle\mathbf{S}(t)\rangle\rangle=\frac{\mathbf{S}_{0}}{3}\left\{1+\frac{2}{\left(1+\sigma^{2} t^{2} / 2\right)^{1 / 2}}\left[1-\left(\frac{1}{1+\sigma^{2} t^{2} / 2}+\sigma^{2} T_{\Delta}^{2}\right)\right.\right. \\
\left.\left.\frac{2}{1+\sigma^{2} t^{2} / 2}\left(\frac{t}{2 T_{\Delta}}\right)^{2}\right] \exp \left(-\frac{1}{1+\sigma^{2} t^{2} / 2}\left(\frac{t}{2 T_{\Delta}}\right)^{2}\right)\right\}
\end{gathered}
$$

Starting from Merkulov's model $(\sigma=0)$, we see in Fig. 4 that in more realistic QD systems, the dip can be washed out by the dot size fluctuations (here $\sigma=1.75 \times 10^{-3} \mathrm{ps}^{-1}$ ). The interest in our approach is to separate the different contributions to the decay of $\left\langle<\mathbf{S}(t)>>\right.$. Note that $\sigma \sim 10^{-3} \mathrm{ps}^{-1}$ corresponds typically to the dots size variations in our samples. The very beginning of the kinetics corresponds to the end of a fast depolarising process that occurs during the electron capture and energy relaxation within the dot, which we have not included in the model. For $t>100 p s$ we can see from Figs. 3 
and 4 that the calculation is in satisfying agreement with the data.

Under longitudinal magnetic field, it is also possible to average the time evolution of the ensemble average spin using the model of Merkulov et al. and taking into account the dots size variations. Using a similar statistical approach, we obtain here:

$$
\begin{gathered}
\langle\langle\mathbf{S}(t)\rangle\rangle=\frac{\mathbf{S}_{0}}{3}\left\{1-\frac{1-\cos \left(\omega_{B} t\right)}{\omega_{B}^{2}} \frac{1}{\left(1+\sigma^{2} t^{2} / 2\right)^{3 / 2}}\right. \\
\left.\left[\sigma^{2}+\frac{1}{T_{\Delta}^{2}\left(1+\sigma^{2} t^{2} / 2\right)}\right] \exp \left(-\frac{1}{1+\sigma^{2} t^{2} / 2}\left(\frac{t}{2 T_{\Delta}}\right)^{2}\right)\right\}
\end{gathered}
$$

where $\omega_{B}=g_{e} \mu_{B} B / \hbar$ is the electron Larmor frequency in the external magnetic field $\mathrm{B}$.

This expression has been used in order to compute the curve displayed in Fig. 4 (with $\mathrm{B}=4 \times \Delta_{B}, \sigma=1.75 \times 10^{-3} \mathrm{ps}^{-1}$ ). We clearly observe the quenching of the spin relaxation by nuclei through the application of a weak external magnetic field.

However, we prefer not to include fits in this paper since there are uncertainties on three parameters: the number of interacting nuclei $N_{L}$, the dot size fluctuations, and the exact composition of the dots (Gallium content). Therefore, we can obtain reasonably good fits, but it is not possible to obtain a unique set of parameters.

\section{HOLE SPIN MEMORY EFFECT IN P-DOPED INAS/GAAS QUANTUM DOTS}

We see in Fig. 2 that the circular polarization is not zero at the end of the radiative recombination; this means that part of the resident holes remain polarized after the emission of a photon. In order to measure this polarization, we have performed a pump-probe like photoluminescence experiment [18]. In this experiment a pulse picker is used to decrease the laser repetition frequency down to $4 \mathrm{Mhz}$. Using an interferometer-like setup, a first pulse, $\left(\sigma^{-}\right)$polarized (the pump) excites the sample followed by a second pulse (the probe) exciting the sample $17.5 \mathrm{~ns}$ later with $\left(\sigma^{+}\right)$polarization (the time delay is much larger than the radiative lifetime of $\sim 1$ ns). The degree of circular polarization of the PL emitted after the probe pulse is analyzed with or without a preceding pump pulse and we detect a remarkable difference between the two cases (Fig. 5). Our interpretation is that the pump pulse polarizes the resident holes in the dots (the spin state is "written") and this polarization remains stable long enough ( $>17.5 \mathrm{~ns})$ for the delayed probe pulse to be able to "read" the spin polarization of these resident holes. This spin memory effect could be observed for temperatures up to $75 \mathrm{~K}$.

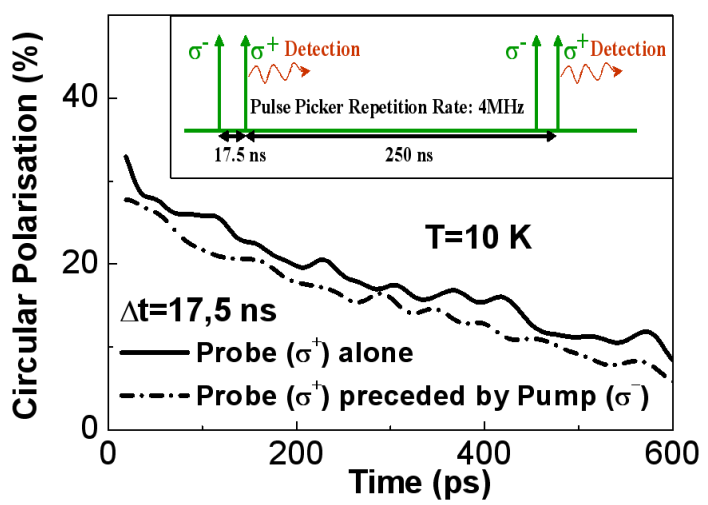

FIG. 5: Circular polarization of the QD luminescence triggered by the $\left(\sigma^{+}\right)$probe preceded or not by the $\left(\sigma^{-}\right)$polarized pump (see text). The time delay between the pump and the probe is $\Delta t=17.5 \mathrm{~ns}$. Note that the horizontal axis represents the time evolution after the arrival of the probe pulse. This probe pulse excites the sample at $t=$ 0 . Inset: Schematic of the pump probe time separation obtained via the use of a pulse picker. The luminescence detection is performed after the probe arrival.

\section{CONCLUSION}

In conclusion, we have investigated the spin dynamics of positively charged excitons in InAs/GaAs quantum dots by time-resolved photoluminescence. We have shown that the dominant electron spin relaxation mechanism at low temperature in QDs is the hyperfine interaction with nuclei. Although this efficient spin relaxation mechanism may strongly limit the performance of future spintronic devices, our measurements show that this spin relaxation can be overcome by applying a magnetic field as small as $100 \mathrm{mT}$, provided for example, by small permanent magnets. In addition, we have observed resident hole spin memory for times as long as $17.5 \mathrm{~ns}$.

\section{Acknowledgement}

We gratefully thank A.E. Zhukov and V.M. Ustinov for the samples growth. We also thank the FSE for the support attributed to P.-F. Braun. Part of this work has been done in the framework of the INTAS project $\mathrm{n}^{o} 2167$.
[1] A. Imamoglu et al., Phys. Rev. Lett. 83, 4204 (1999).

[2] R. Fiederling et al., Nature 402, 787 (1999).

[3] M. Oestreich, Nature 402, 735 (1999), H. Ohno, Science 291, $840(2001)$
[4] A. Imamoglu et al., Phys. Rev. Lett. 91, 17402 (2003).

[5] L. M. Woods et al., Phys. Rev. B 66, R161318 (2002).

[6] I. A. Merkulov et al., Phys. Rev. B 65, 205309 (2002).

[7] A. Khaetskii et al., Phys. Rev. Lett. 88, 186802 (2002). 
[8] Y. Semenov et al., Phys. Rev. B 67, 73301 (2003).

[9] D. Gammon et al., Science 277, 87 (1997).

[10] J. A. Gupta et al., Phys. Rev. B 59, 10421 (1999).

[11] I. Ignatiev et al., Physica E 17, 361 (2003).

[12] R. Dzhioev et al., Phys. Sol. State 41, 2014 (1999).

[13] M. Paillard et al., Phys. Rev. Lett. 86, 1634 (2001).

[14] A. S. Lenihan et al., Phys. Rev. Lett. 88, 223601 (2002).

[15] E. Tsitishvili et al., Phys. Rev. B 66, 161405 (2002).

[16] T. Amand et al., Superlattices and Microstructures 32, 157 (2002).

[17] T. Flissikowski et al., Phys. Rev. B 68, 161309 (2003).

[18] S. Cortez et al., Phys. Rev. Lett 89, 207401 (2002).

[19] M. Kroutvar et al., Nature 432, 81 (2004).

[20] K. Gundogdu et al., Appl. Phys Lett. 84, 2793 (2004).

[21] M. Bayer et al., Phys. Rev. Lett. 82, 1748 (1999).

[22] S. I. Erlingson et al., Phys. Rev. B 64, 195306 (2001).

[23] R. J.Warburton et al., Nature (London) 405, 926 (2000).

[24] E. I. Gryncharova et al., Sov. Phys. Semicond. 11, 997 (1977).

[25] A. Bracker et al., Phys. Rev. Lett. 94, 047402 (2005).

[26] S. Laurent et al., Acta Physica Polonica A 106, 185 (2004).

[27] P.-F. Braun et al., Phys. Rev. Lett. 94, 116601 (2005).

[28] T. Flissikowski et al., Phys. Rev. Lett 86, 3172 (2001).
[29] W. Langbein et al., Phys. Rev. B 69, 161301 (2004), A. Tartakovskii et al., Phys. Rev. Lett. 93, 57401 (2004).

[30] T. Damen et al., Phys. Rev. Lett. 67, 3432 (1991).

[31] This plateau value is due to the fact that no relaxation occurs when the nuclear field component is oriented along the initial electron spin $\mathbf{S}_{0}$.

[32] In contrast, the decay of the components of the ensemble average spin polarization perpendicular to the applied magnetic field cannot be suppressed.

[33] R. I. Dzhioev et al., Phys. Rev. B 66, 245204 (2002).

[34] J. S. Colton et al., Phys. Rev. B 69, 121307 (2004).

[35] We did not observe any significant difference, within our experimental accuracy, in the spin dynamics at $\mathrm{Bz} \neq 0$ for a $\sigma^{+}$or $\sigma^{-}$polarized excitation.

[36] K. V. Kavokin, Phys. Stat. Sol. (a) 195, 592 (2003).

[37] S. Laurent et al., PhD thesis, University Paris VI (2004), http://tel.ccsd.cnrs.fr.

[38] D. Paget et al., Phys. Rev. B 15, 5780 (1977).

[39] M. Paillard et al., Appl. Phys. Lett. 76, 76 (2000).

[40] The same calculation for GaAs dots of the same size yields $T_{\Delta}^{\text {GaAs }} \approx 2550 \pm 950 \mathrm{ps}$. 